Tunable coaxial cavity resonator for linear and nonlinear microwave characterization of superconducting wires

This article has been downloaded from IOPscience. Please scroll down to see the full text article.

2011 Supercond. Sci. Technol. 24095008

(http://iopscience.iop.org/0953-2048/24/9/095008)

View the table of contents for this issue, or go to the journal homepage for more

Download details:

IP Address: 147.163.21.165

The article was downloaded on 07/09/2011 at 15:54

Please note that terms and conditions apply. 


\title{
Tunable coaxial cavity resonator for linear and nonlinear microwave characterization of superconducting wires
}

\author{
A Agliolo Gallitto, G Bonsignore, M Li Vigni and A Maccarone \\ CNISM and Dipartimento di Fisica, Università di Palermo, Via Archirafi 36, I-90123 Palermo, \\ Italy
}

Received 20 April 2011, in final form 13 June 2011

Published 29 July 2011

Online at stacks.iop.org/SUST/24/095008

\begin{abstract}
We discuss experimental results obtained using a tunable cylindrical coaxial cavity resonator constituted by an outer $\mathrm{Cu}$ cylinder and an inner bulk $\mathrm{Bi}_{1.8} \mathrm{~Pb}_{0.26} \mathrm{Sr}_{2} \mathrm{Ca}_{2} \mathrm{Cu}_{3} \mathrm{O}_{10+x}$ cylinder. We have used this device to investigate the microwave response of the superconducting wire, both in the linear and nonlinear regimes. In particular, by tuning the different modes of the cavity to make them resonant at exactly harmonic frequencies, we have detected the power emitted by the superconductor at the second- and third-harmonic frequency of the driving field. The results obtained in the nonlinear regime, whether for the microwave surface impedance or the harmonic emission, are discussed in the framework of the models reported in the literature for granular superconductors. The use of this kind of device could be of great interest to investigate and characterize wires of large dimensions, to be used for implementing superconducting-based microwave devices.
\end{abstract}

\section{Introduction}

Superconducting materials, due to their low microwave (MW) surface impedance, are preferred to normal metals for assembling MW devices, such as filters, antennas, resonators, etc [1-4]. Investigation of the MW response of superconductors (SC), besides its importance for basic physics, gives information on specific properties of the investigated sample, allowing us to recognize the materials suitable for designing devices operating at MW frequencies [3, 4]. Since the discovery of high- $T_{\mathrm{c}}$ superconductors (HTSC), several studies have been carried out to exploit the potential of these SC for implementing MW devices; comprehensive reviews on MW applications of HTSC have been reported in [1-3].

Actually, the main factor limiting the use of HTSC in passive MW devices is the occurrence of nonlinear effects at high input power levels. Nonlinear effects manifest themselves in the power dependence of the surface impedance [5-9], intermodulation product [5, 8-10] and harmonic generation [5, 11-13], which worsen the performance of passive MW devices; so, it is important to estimate the power and/or field values at which nonlinearity comes into play, as well as to understand its physical origin.

One of the MW devices mostly used in both applications and basic-physics studies of SC is the resonant cavity. From the technological point of view, superconducting resonant cavities can be conveniently used in all the systems requiring high selectivity in the signal frequency. In contrast, the cavityperturbation technique allows measuring the MW surface impedance, $Z_{\mathrm{s}}=R_{\mathrm{s}}+\mathrm{i} X_{\mathrm{s}}$, of superconducting materials, which is the most important property characterizing the MW response [14-17]. With the advance of the modern techniques for film deposition, planar transmission line filters or stripline resonators of small size can be fabricated and used in many applications. Despite the availability of these smaller size filters, bulk- or coated-cavity filters provide higher quality factor and reduced nonlinear effects. This is so because small cross-section areas for current flow in films lead to high current densities, even at relatively low input power levels, enhancing nonlinear effects $[9,18]$. Because of these properties, superconducting films have been recommended for implementing MW power limiters [19]. Bulk-cavity filters are preferred in all the applications in which miniaturization is not important [20], such as, for example, high-frequency wireless communication systems, satellite transmission systems, radars and high-power transmission lines.

Characterization at MW frequencies of SC is generally performed by locating a small sample in resonant cavities operating at fixed frequencies. To investigate the surface impedance, one measures the variations of the resonant 
frequency and the quality factor of the cavity induced by the sample [14]. The intermodulation product is investigated by applying two MW signals, whose frequencies are within the bandwidth of the cavity, and detecting third-order intermodulation frequencies by a spectrum analyzer [9]. Harmonic generation is investigated by using a nonlinear MW spectrometer, whose basic element is the bimodal cavity, resonating at two different frequencies $(\omega$ and $n \omega)[13,21]$; different cavities have to be used for detecting the different $n$ th-order harmonic signals. So, for a complete MW characterization different set-ups and cavities are necessary.

A coaxial cavity resonator with a superconducting inner conductor has been proposed to conveniently measure the frequency dependence of the MW surface resistance, $R_{\mathrm{S}}$ [1]. Prototypes of coaxial resonators have been built using normal metal as an outer conductor and HTSC as an inner conductor and used for measuring the MW surface resistance of the inner SC [22-24]. The use of this kind of resonator is particularly suitable for characterizing samples with dimensions too large for the cavity-perturbation method. The inner conductor can be constituted by bulk or coated slabs and/or wires. The length can be dimensioned to investigate different frequency ranges. High sensitivity can be achieved by using thin central wires or SC materials for both the inner and the outer cylinders. However, for investigating the MW properties of SC as a function of a DC magnetic field, the outer cylinder should be made of normal metal. In this paper, we discuss the potential of such a type of device for a complete investigation of SC in both the linear and nonlinear regimes. We have built a tunable cylindrical coaxial cavity constituted by an outer $\mathrm{Cu}$ cylinder and an inner bulk $\mathrm{Bi}_{1.8} \mathrm{~Pb}_{0.26} \mathrm{Sr}_{2} \mathrm{Ca}_{2} \mathrm{Cu}_{3} \mathrm{O}_{10+x}$ cylinder and have used it for different types of measurements. In the linear regime, we have measured $R_{\mathrm{S}}$ of the inner $\mathrm{SC}$ as a function of the frequency, at zero DC magnetic field. In the nonlinear regime, we have measured the power dependence of the surface impedance and the signals emitted by the SC at the second- and third-harmonic frequencies of the driving field. In order to detect the harmonic signals, the different modes of the cavity have been tuned to make them resonant at exactly harmonic frequencies. This has been done by inserting two metallic screws in the outer conductor, which modify the impedance of the whole cavity and slightly change the resonant frequencies. This allows one to measure all the nonlinear properties of the investigated material in the same excitation conditions.

\section{Coaxial cavity resonator: theoretical aspects}

A coaxial cavity resonator consists of an outer conductor tube containing an inner wire. An extensive discussion of this MW device is reported in [1]; here, we discuss only the essential concepts useful for understanding the present work. Coaxial resonator supports TEM, TM and TE modes; however, the design of our cavity is such that, in the investigated frequency range (1-13 GHz), the only detectable modes are the TEM ones, corresponding to standing waves in which an integer number of half-wavelengths nearly matches the length of the inner conductor. In these modes, electric-field lines are radial and magnetic-field lines wind around the central conductor; in an open-circuit-end resonator, as is our cavity, different TEM modes can be fed, with the condition that at both the innerconductor ends the electric field is maximum and the magnetic field is zero. The quality factor of a coaxial cavity with opencircuit ends, operating in the TEM mode, is given by

$$
Q_{\mathrm{c}}=\frac{\omega \mu_{0} \ln (b / a)}{\frac{R_{\mathrm{s} a}}{a}+\frac{R_{\mathrm{s} b}}{b}},
$$

where $a$ is the radius of the inner conductor, $b$ is the inner radius of the outer conductor, $R_{\mathrm{s} a}$ and $R_{\mathrm{s} b}$ are the surface resistances of the inner and outer conductors, respectively, and $\omega$ is the angular frequency of the considered mode.

Equation (1) includes only the energy losses occurring in the walls of the outer and inner conductors; if the cavity is filled with a dielectric medium, the unloaded quality factor of the resonator, $Q_{\mathrm{U}}$, is affected by conductor losses and dielectric losses and is given by

$$
\frac{1}{Q_{\mathrm{U}}}=\frac{1}{Q_{\mathrm{c}}}+\frac{1}{Q_{\mathrm{d}}}
$$

where $Q_{\mathrm{d}}$ takes into account the dielectric losses; when the dielectric completely fills the cavity, $1 / Q_{\mathrm{d}}=\tan \delta$.

Moreover, since the cavity is generally coupled to an external circuit through an excitation and a detection line, additional energy losses out of the coupling ports occur and the measured (loaded) quality factor, denoted as $Q_{\mathrm{L}}$, differs from $Q_{\mathrm{U}}$. If the cavity is weakly coupled to the external circuit, only small corrections have to be done to calculate $Q_{\mathrm{U}}$; in any case, this correction can be easily calculated considering the coupling coefficients (see chapter 4 of [1]).

From equations (1) and (2), the MW surface resistance of the inner conductor can be written as

$$
R_{\mathrm{s} a}=\Gamma\left[\frac{1}{Q_{\mathrm{U}}}-\tan \delta\right]-\frac{a}{b} R_{\mathrm{s} b},
$$

where $\Gamma$ is the geometry factor, given by

$$
\Gamma=a \omega \mu_{0} \ln (b / a) .
$$

From equation (3), one can see that to determine $R_{\mathrm{s} a}$ it is necessary to know $R_{\mathrm{s} b}$ and $\tan \delta$.

\section{The tunable coaxial cavity}

The MW coaxial cavity consists of a copper outer tube, of length $h=43.8 \mathrm{~mm}$ and inner diameter $2 b=8.1 \mathrm{~mm}$, with the dielectric insulator PTFE supporting an inner bulk $\mathrm{Bi}_{1.8} \mathrm{~Pb}_{0.26} \mathrm{Sr}_{2} \mathrm{Ca}_{2} \mathrm{Cu}_{3} \mathrm{O}_{10+x}$ ( $\mathrm{Pb}-\mathrm{BSCCO}$ ) cylinder, of length $l=40 \mathrm{~mm}$ and diameter $2 a=3 \mathrm{~mm}$. The $\mathrm{Pb}-$ BSCCO cylinder has been produced by Can Superconductors s.r.o. using a basic powder isostatically pressed into its shape by $0.7 \mathrm{GPa}$ and subsequently thermally treated at $837^{\circ} \mathrm{C}$ in air. The basic powder is prepared by the reaction in the solid phase of corresponding volumes of oxides of bismuth, lead and strontium, and calcium carbonates. Further material 


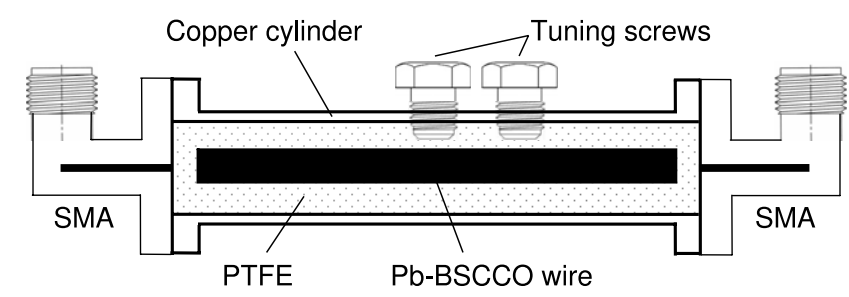

Figure 1. Schematic diagram of the coaxial cavity.

characterization can be found on the web site of the company. The cavity is used in the transmission mode; it is coupled to the external excitation and detection lines by a capacitive gap at both ends of the resonator. The gap between the SC and the feed wire is adjusted to give weak coupling, so that measurements of $Q_{\mathrm{L}}$ require only small corrections to calculate $Q_{\mathrm{U}}$. Two small plates of PTFE are also located at the ends of the $\mathrm{Pb}-\mathrm{BSCCO}$ cylinder, in order to prevent intermittent electrical contact. A schematic view of the cavity is shown in figure 1.

Because of the capacitive effects at the gaps, the electromagnetic field extends slightly beyond the ends of the inner SC. This causes small differences of the resonant frequencies from the expected ones; for the same reason, the resonant frequencies of the higher frequency modes are not exactly harmonics of the fundamental one. In order to tune the first three modes of the cavity and make them resonant at exactly harmonic frequencies, we have made two holes in the outer conductor for inserting two brass screws that, modifying the impedance of the whole cavity, slightly change the resonant frequencies. As shown in figure 1, a screw is located in proximity of the middle length of the outer conductor; by regulating the penetration of this screw into the cavity, we can tune the first two modes within the frequency bandwidth. A second screw, located at about one-third of the length, allows us to tune the first and third modes as well. This procedure is done to feed the cavity in the fundamental mode and detect the second-harmonic (SH) and third-harmonic (TH) signals emitted by the SC wire. We remark that the length of penetration of the screws into the cavity does not appreciably affects the quality factor and the coupling coefficients of the cavity with the external circuit.

The cavity has been used to perform several kinds of measurements. $R_{\mathrm{S}}$ of the $\mathrm{Pb}-\mathrm{BSCCO}$ has been measured at fixed temperatures (below $T_{\mathrm{c}}$ ) as a function of the frequency and input power level. Furthermore, we have investigated the signals emitted by the $\mathrm{SC}$ at the $\mathrm{SH}$ and $\mathrm{TH}$ frequencies of the driving field. In order to make measurements as a function of the external magnetic field, the cavity is placed between the poles of an electromagnet that generates DC magnetic fields up to $\mu_{0} \boldsymbol{H}_{\mathbf{0}} \approx 1 \mathrm{~T} ; \boldsymbol{H}_{\mathbf{0}}$ can be rotated in the plane containing the cylinder axis to change its orientation with respect to the MW magnetic field. Two additional coils, independently fed, allow us to compensate the residual magnetic field and making measurements at low magnetic fields.

\section{Microwave surface resistance}

\subsection{Measurement technique}

As one can see from equation (3), in order to determine $R_{\mathrm{s} a}$ of the $\mathrm{Pb}-\mathrm{BSCCO}$ wire, we need to know $Q_{\mathrm{U}}$, the MW surface resistance of the outer conductor, $R_{\mathrm{s} b}$, and $\tan \delta$. At low input power $\left(P_{\text {in }} \lesssim 0 \mathrm{dBm}\right), Q_{\mathrm{L}}$ has been measured using an HP8719D network analyzer (NA), operating in the frequency range $50 \mathrm{MHz}-13.5 \mathrm{GHz}$, and detecting the frequency response of the transmitted wave. $Q_{\mathrm{U}}$ has been determined by taking into account the coupling coefficients, $\beta_{1}$ and $\beta_{2}$, for both the external lines; these coefficients can be calculated by directly measuring the reflected power at each line, as described in [1], chapter 4. $Q_{\mathrm{U}}$ is given by $Q_{\mathrm{U}}=\left(1+\beta_{1}+\beta_{2}\right) Q_{\mathrm{L}}$.

To determine the frequency dependence of $R_{\mathrm{s} a}$, we have previously calibrated the cavity using an inner copper cylinder having the same dimension of the $\mathrm{Pb}-\mathrm{BSCCO}$ one. At a fixed frequency, and in the absence of PTFE, we have determined the surface resistance of the copper from equation (1) letting $R_{\mathrm{s} a}=R_{\mathrm{s} b}$. Subsequently, we have investigated the frequency response of the cavity with the dielectric insulator PTFE supporting the inner copper cylinder, and measured the quality factor and the central frequency in the different resonant modes. By assuming the $\sqrt{\omega}$ law for the frequency dependence of $R_{\mathrm{s} b}$, we have determined $\tan \delta$ of the PTFE as a function of the frequency. We have obtained $\tan \delta \sim 10^{-4}$, slowly increasing with the frequency. Once $Q_{\mathrm{U}}(f), R_{\mathrm{s} b}(f)$ and $\tan \delta(f)$ are known, from equation (3) we obtain the frequency dependence of the MW surface resistance of the SC in the frequency range in which the different resonant modes fall.

Measurements of the MW surface impedance as a function of the maximum value of the MW magnetic field, $H_{\mathrm{MW}}$, have been performed varying the input power levels and using two different MW sources, for the fundamental mode and the higher frequency modes. For the fundamental mode $(f=$ $\omega / 2 \pi \approx 2.525 \mathrm{GHz}$ ), the continuous wave generated by the NA is modulated to obtain a train of MW pulses, with pulse width $\approx 10 \mu$ s and pulse repetition rate $100 \mathrm{~Hz}$. The pulsed signal is amplified up to a peak power level of $\approx 44 \mathrm{dBm}$ and driven into the cavity through the excitation line. The transmitted signal is detected by a solid-state diode. The resonance curve of the cavity is reproduced by sweeping the continuous-wave frequency of the NA. By a Lorentzian fit of the resonance curve, we extracted the central frequency and the unloaded quality factor of the cavity. From $Q_{\mathrm{U}}$, we have determined the surface resistance of the sample as discussed above; from the central frequency, we have determined the surface reactance, $X_{\mathrm{s}}$. The surface reactance can be determined by measuring the shift of the resonance frequency of the cavity, apart from an additive constant $X_{0}$. To obtain $X_{\mathrm{s}}$ in absolute units, it is necessary to know $X_{0}$, which can be determined by imposing the condition of the normal skin effect at $T=T_{\mathrm{c}}$. Since in the normal state the resonance curve is broadened and deformed, we will report only the variation of $X_{\mathrm{s}}$ with respect to that obtained at the lowest MW magnetic field, which is given by

$$
\Delta X_{\mathrm{s}} \equiv X_{\mathrm{s}}\left(H_{\mathrm{MW}}\right)-X_{\mathrm{s}}(0)=-2 \Gamma \frac{f\left(H_{\mathrm{MW}}\right)-f(0)}{f(0)} .
$$




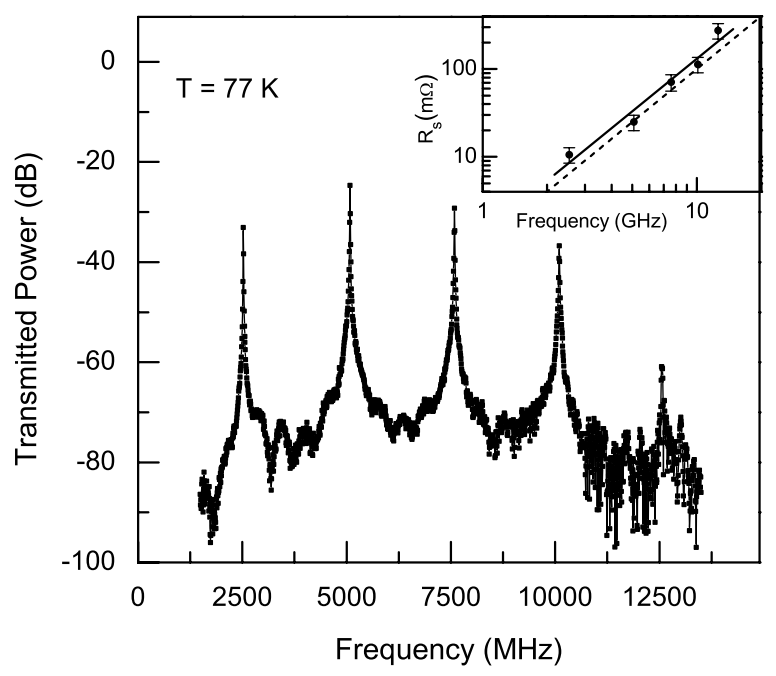

Figure 2. Transmitted power over input power through the coaxial cavity as a function of the frequency. Inset: frequency dependence of $R_{\mathrm{s}}$ of the inner $\mathrm{Pb}-\mathrm{BSCCO}$ cylinder; the continuous line is the best-fit curve of data, while the dashed one represents the $f^{2}$ law.

The values of the $H_{\mathrm{MW}}$ have been calculated considering the input power, the unload quality factor of the cavity and the coupling coefficients in the different modes, following chapter 3 of [1].

For the higher frequency modes, since we do not dispose of the MW amplifier at frequencies higher than $4 \mathrm{GHz}$, we have used a MW sweeper that generates a continuous wave up to power levels of $\approx 20 \mathrm{dBm}$. Also in this case, the resonance curve of the cavity is reproduced by sweeping the continuouswave frequency.

\subsection{Results and discussion}

Figure 2 shows the MW response of the coaxial cavity resonator with the bulk $\mathrm{Pb}-\mathrm{BSCCO}$ wire in the frequency range $1-13 \mathrm{GHz}$, at $T=77 \mathrm{~K}$ and $P_{\text {in }} \approx-1 \mathrm{dBm}$. The inset shows the frequency dependence of $R_{\mathrm{S}}$ of the $\mathrm{Pb}-\mathrm{BSCCO}$ wire at the same temperature and input power level. The values of $R_{\mathrm{S}}$ are consistent with those reported in the literature for HTSC ceramic samples [23-25]. The continuous and the dashed lines in the inset show the best-fit curve of the data and the $f^{2}$ law, respectively.

The $f^{2}$ dependence of $R_{\mathrm{S}}$ is expected in the London twofluid model as well as in the BCS theory when the imaginary component of the complex conductivity is much larger than the real one. It has been experimentally shown that it is also valid in HTSC single crystals and high quality thin films. For bulk HTSC materials, it has been observed that $R_{\mathrm{S}} \propto f^{n}$ with $n$ ranging from 1 to 2 , depending on the investigated sample $[22,24,26,27]$. Deviations from the $f^{2}$ law have been ascribed to normal material inclusion at the grain boundaries, poor surface quality, wide variation of crystal-axis orientation of the grains, etc [26, 27]. On the other hand, models developed to describe the electromagnetic response of weakly coupled grains have shown that different $f$ dependences are expected in the different field and frequency ranges [28]. In particular,

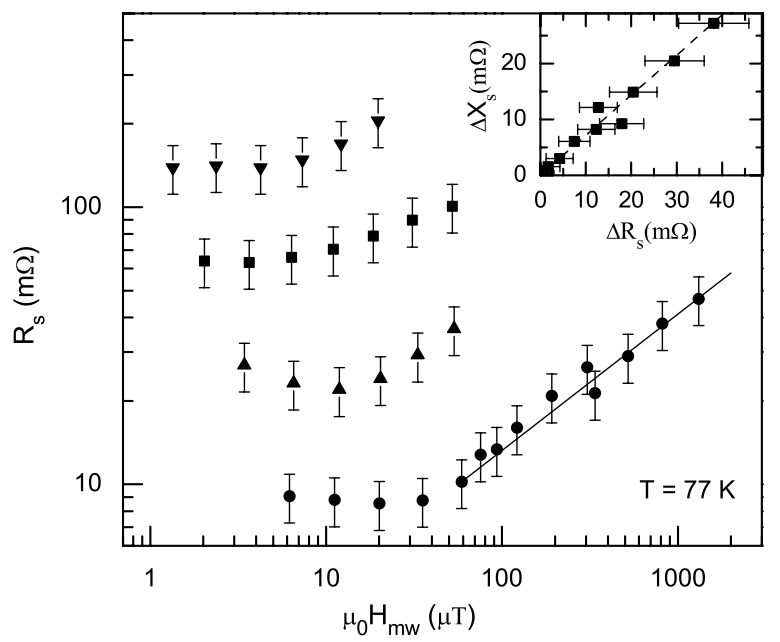

Figure 3. $R_{\mathrm{S}}$ of the $\mathrm{Pb}-\mathrm{BSCCO}$ wire as a function of the maximum value of the MW magnetic field, at the resonant frequencies of the different TEM modes: $f \approx 2.5 \mathrm{GHz}(\bullet) ; f \approx 5.1 \mathrm{GHz}(\boldsymbol{\Delta})$; $f \approx 7.6 \mathrm{GHz}(\boldsymbol{\square}) ; f \approx 10.1 \mathrm{GHz}(\boldsymbol{\nabla})$. The uncertainties in $R_{\mathrm{s}}$ $(\approx 20 \%)$ are essentially due to the determination of $\tan \delta$ of PTFE. The inset shows the variations of $X_{\mathrm{s}}$ as a function of the variations of $R_{\mathrm{s}}$, obtained at different values of $H_{\mathrm{MW}}$, for $f \approx 2.5 \mathrm{GHz}$.

an $f^{2}$ law is expected at MW magnetic fields lower than the Josephson lower critical field, $H_{\mathrm{c} 1 j}$. To our knowledge, results on bulk BSCCO samples are not reported in the literature; most investigations concern YBCO samples. The data of figure 2 have been obtained with $\mu_{0} H_{\mathrm{MW}}<10 \mu \mathrm{T}$; so, the nearly quadratic frequency dependence of $R_{\mathrm{S}}$ we obtained can be ascribed to the fact that $H_{\mathrm{MW}}<H_{\mathrm{c} 1 j}$.

Figure 3 shows the MW surface resistance of the $\mathrm{Pb}-$ BSCCO wire as a function of the maximum value of the MW magnetic field inside the cavity at the resonant frequencies of different TEM modes. As one can see, above a threshold value of $H_{\mathrm{MW}}$, slightly depending on the frequency, $R_{\mathrm{S}}$ deviates from its initial value, highlighting the occurrence of nonlinear effects. The MW amplifier operating at $f \sim 2.5 \mathrm{GHz}$ allowed us to investigate a wide range of MW fields; the largest value of $H_{\mathrm{MW}}$ in the figure corresponds to an effective input power level in the cavity of the order of $37 \mathrm{dBm}$. At this frequency, nonlinear effects set in for $\mu_{0} H_{\mathrm{MW}} \gtrsim 40 \mu \mathrm{T}$; the data above this threshold have been fitted by an $H_{\mathrm{MW}}^{n}$ law, while the continuous line in the figure shows the best-fit curve obtained with $n=0.63$. The inset is a plot of $\Delta X_{\mathrm{S}} \equiv X_{\mathrm{s}}\left(H_{\mathrm{MW}}\right)-X_{\mathrm{s}}(0)$ as a function of $\Delta R_{\mathrm{S}} \equiv R_{\mathrm{S}}\left(H_{\mathrm{MW}}\right)-R_{\mathrm{S}}(0)$, where $X_{\mathrm{s}}(0)$ and $R_{\mathrm{S}}(0)$ are the values of the MW surface reactance and resistance, respectively, obtained at the lowest $H_{\mathrm{MW}}$ value. The dashed line in the inset is the linear fit of the data of slope 0.73 .

The nonlinear behavior of the MW surface impedance in HTSC has been investigated by several authors especially in films [8, 9]; only a few investigations concern bulk samples [22, 24]. In spite of considerable effort, an universal model able to explain all the observed features has not yet been developed. Reviews of the results on the nonlinear MW surface impedance and the proposed models have been discussed in $[5,6,8,28]$. A common characteristic is that for $H_{\mathrm{MW}}<H_{\mathrm{cl} j}, R_{\mathrm{S}}$ varies quadratically with the frequency 
and the field-induced $Z_{\mathrm{s}}$ variations are small and not easily detectable $[8,28]$. For $H_{\mathrm{MW}}>H_{\mathrm{cl} j}$, all nonlinear mechanisms are described with power dependences of $\Delta R_{\mathrm{S}} \propto H_{\mathrm{MW}}^{\alpha}$ and $\Delta X_{\mathrm{s}} \propto H_{\mathrm{MW}}^{\alpha}$, with $0.5 \leqslant \alpha \leqslant 2$. Among the various models, some of them are based on the so-called coupledgrain RSJ network (CG-RSJ) [29, 30]; others describe the nonlinearity of $Z_{\mathrm{s}}$ considering dynamics of Josephson fluxons in grain boundaries [28]. It has been suggested that, in order to recognize the process responsible for the field-induced variations of $Z_{\mathrm{s}}$, an useful parameter is $r \equiv \Delta X_{\mathrm{s}} / \Delta R_{\mathrm{s}}$, though in some cases no correlation between $R_{\mathrm{s}}$ and $X_{\mathrm{s}}$ has been observed [8].

Considering the Josephson-fluxon dynamics, different results are expected depending of the motion regime: intergrain critical state gives $1.5 \leqslant r \leqslant 2.5$, depending on the geometry; motion of fluxons induced by the MW current under pinning and viscous-drag forces gives $r=\omega_{0 j} / \omega$, where $\omega_{0 j}$ is the depinning frequency and $\omega$ the working frequency, for $\omega<$ $\omega_{0 j}$ and $r \approx 1$ for $\omega>\omega_{0 j}$ [28].

In the framework of the CG-RSJ models, the material is modeled as a series network of superconducting grains connected by resistively shunted Josephson junctions (RSJ). An analytical expression can be obtained considering the complex impedance of the RSJ and assuming a single type of junction behavior for the whole sample [29]. A more realistic extension of the CG-RSJ model has been introduced by Herd et al [30] to include distributions of $I_{\mathrm{c}}$ and $R_{n}$, where $I_{\mathrm{c}}$ is the critical current of the junction and $R_{n}$ is its resistance in the normal state. Numerical calculations performed considering a wide Gaussian distribution of $I_{\mathrm{c}} R_{n}$ give $r \simeq 0.7$.

From the fit of the data reported in the inset of figure 3 , we obtain $r=0.73 \pm 0.13$; this finding suggests that our results can be justified in the framework of the extended CGRSJ model. However, since the obtained $r$ value is near unity, we cannot rule out the possibility that a description in terms of fluxon motion along grain boundaries accounts for the observed $Z_{\mathrm{s}}$ nonlinearity if the depinning frequency of intergrain fluxons in our sample is of a few GHz.

\section{Harmonic generation}

\subsection{Measurement technique}

In order to detect the power emitted by the bulk $\mathrm{Pb}-\mathrm{BSCCO}$ cylinder at the $\mathrm{SH}$ and $\mathrm{TH}$ frequencies of the driving field, it is necessary to properly move the two brass screws to tune the first and second resonant modes of the cavity and/or the first and the third ones. To do these measurements we have used the following procedure. The continuous wave generated by the NA at the frequency of the fundamental mode is modulated to obtain a train of MW pulses, with pulse width $5 \mu$ s and pulse repetition rate $100 \mathrm{~Hz}$, and amplified up to a peak power level of $\approx 44 \mathrm{dBm}$. The pulsed wave is filtered by a low-pass filter, reducing any harmonic content by more than $60 \mathrm{~dB}$, and feeds the fundamental mode of the cavity through the excitation line. The wave coming from the cavity through the detection line, which contains components oscillating at harmonic frequencies because of the nonlinear

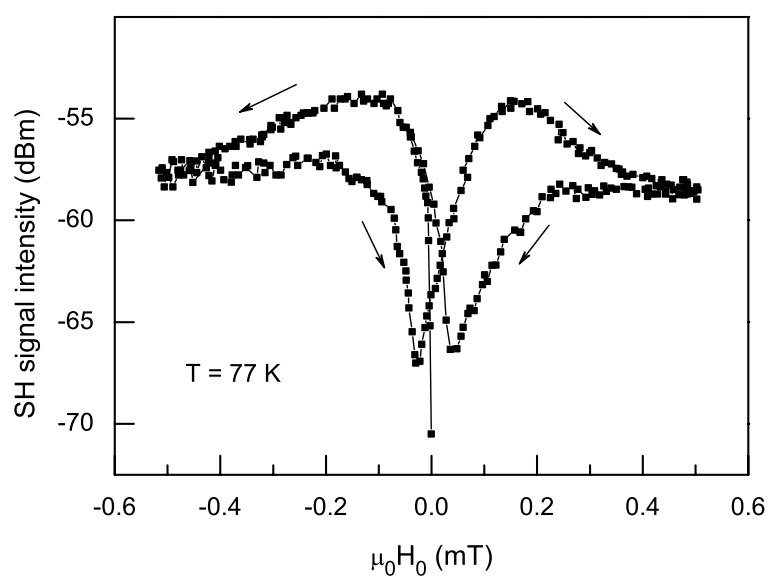

Figure 4. SH signal intensity as a function of the DC magnetic field, $H_{0}$. The effective input peak power at $2.5 \mathrm{GHz}$ is $P_{\text {eff }} \approx 20 \mathrm{dBm}$. The arrows indicate the field-sweep direction.

response of the $\mathrm{SC}$, is filtered by a band-pass filter, with more than $60 \mathrm{~dB}$ rejection at the fundamental frequency, and sent to a superheterodyne receiver. The latter is equipped with a $30 \mathrm{MHz}$ logarithmic amplifier that provides an output voltage proportional to the harmonic power (noise level $\approx-75 \mathrm{dBm}$ ). The signal is displayed by an oscilloscope and recorded on a computer by an IEEE-488 interface, which allows one to automatically acquire the experimental data.

\subsection{Results and discussion}

The SH signal intensity as a function of the DC magnetic field, $H_{0}$, is shown in figure 4; the measurements have been performed at $T=77 \mathrm{~K}$. The effective input peak power in the cavity at the fundamental mode, resonating at $f \sim 2.5 \mathrm{GHz}$, is $P_{\text {eff }} \approx 20 \mathrm{dBm}$; it has been determined by taking into account the coupling coefficient of the excitation line after tuning the first and second modes. We have indicated as $\mathrm{SH}$ signal intensity the effective power at $2 f$ inside the cavity, which has been calculated from the detected power properly rescaled by taking into account the coupling coefficient of the detection line. Since no SH emission is expected for MW magnetic fields perpendicular to $H_{0}$, the DC magnetic field has been oriented perpendicularly to the cylinder axis. By changing the orientation of $H_{0}$, we have verified that the SH signal intensity significantly decreases when the component of $H_{0}$ perpendicular to the cylinder axis decreases. However, a very weak SH signal is also observed for $H_{0}$ parallel to the axis; this may be due to a little misalignment. The SH signal intensity shows a magnetic hysteresis having a butterfly-like shape, which has been already observed in inhomogeneous SC, whether at MW frequencies [13, 31, 32] or at lower frequencies [33-35].

Measurements performed sweeping $H_{0}$ in different ranges from $-H_{\max }$ to $+H_{\max }$ have shown that the hysteresis appears for $\mu_{0} H_{\max } \approx 0.2 \mathrm{mT}$; on increasing $H_{\max }$, the amplitude of the hysteresis loop increases and the sharp minima at low fields become less pronounced until they almost completely disappear for $\mu_{0} H_{\max } \gtrsim 2 \mathrm{mT}$. Moreover, measurements 


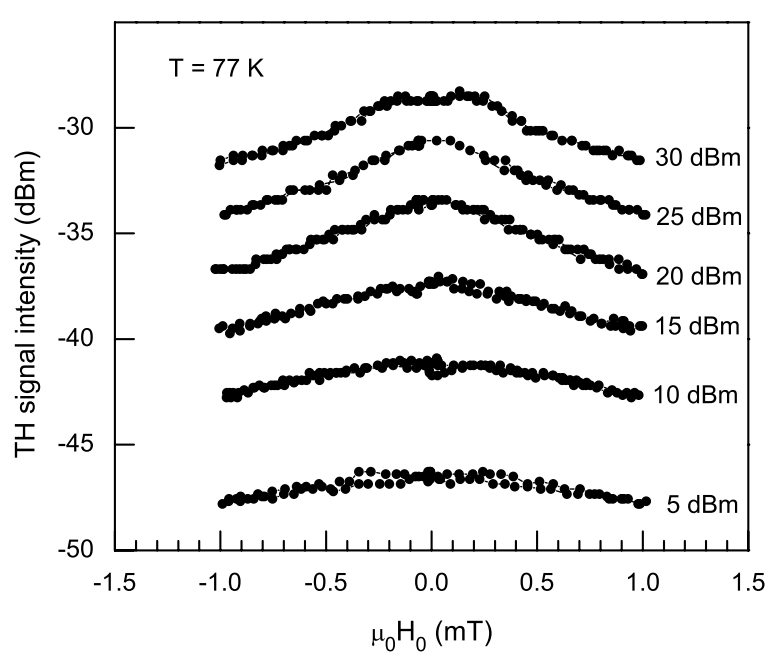

Figure 5. TH signal intensity as a function of the DC magnetic field, at different power levels (the values indicated on the right-hand side of the plot refer to the effective input peak power in the cavity at the resonant frequency of the fundamental mode).

performed at different input power levels have shown that the features of the $\mathrm{SH}$ versus $H_{0}$ curves do not exhibit noticeable variations.

Figure 5 shows the TH signal intensity as a function of the DC magnetic field, at different $P_{\text {eff }}$ values at the fundamental mode. In order to compare the $\mathrm{SH}$ and $\mathrm{TH}$ response in the same field geometry, $H_{0}$ is perpendicular to the cylinder axis. By varying the DC field orientation, the features of the $\mathrm{TH}$ versus $H_{0}$ curves do not significantly change; only an increase of the signal intensity of about $1 \mathrm{~dB}$ is observed for $H_{0}$ parallel to the cylinder axis. Measurements performed for different $H_{\text {max }}$ values have shown that the TH versus $H_{0}$ curve exhibits a monotonic decrease with $H_{0}$ and no hysteresis is observed until $\mu_{0} H_{\max }$ reaches values larger than $2 \mathrm{mT}$.

By comparing the results of figures 4 and 5, one can note that the TH signal is much more intense than the $\mathrm{SH}$ one. As an example, the intensities of the SH and TH signals, generated with effective input peak power at the fundamental mode of $20 \mathrm{dBm}$, differ by about $20 \mathrm{~dB}$.

Figure 6 shows the field dependence of both $\mathrm{SH}$ and $\mathrm{TH}$ signal intensity, obtained at $T=77 \mathrm{~K}$ by sweeping $\mu_{0} H_{0}$ from zero to $5 \mathrm{mT}$ and back. In the $\mathrm{SH}$ signal, the hysteresis is enhanced, extends in a wide field range, and the sharp minima in the decreasing-field branch are no longer present. In contrast, only a small hysteresis is observed in the TH signal, which extends in a narrow field range. The large difference in the $\mathrm{SH}$ and $\mathrm{TH}$ signal intensities is still present and enhanced (at high fields); this is so because the $\mathrm{SH}$ signal reduces faster with the field than the TH one. We would like to remark that it was possible to highlight this peculiarity of the nonlinear MW emission because we used the same device (with the same excitation conditions) to detect both the $\mathrm{SH}$ and $\mathrm{TH}$ emission.

Harmonic emission has been investigated in both conventional and high- $T_{\mathrm{c}} \mathrm{SC}$; several mechanisms have been recognized and they play a different role depending on temperature, applied magnetic field, frequency and type of $\mathrm{SC}[5,6,12]$. In any case, harmonic distortion is a

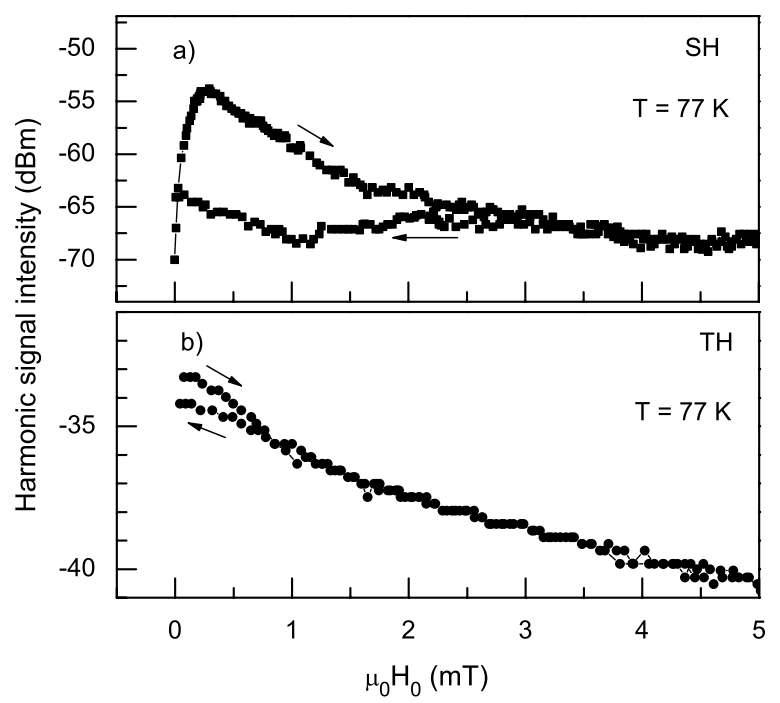

Figure 6. (a) $\mathrm{SH}$ and (b) TH signal intensity as a function of the DC magnetic field; $P_{\text {eff }} \approx 20 \mathrm{dBm}$.

peculiarity of the superconducting state; harmonic signals disappear whenever the SC goes into the normal state. It is widely accepted that, at temperatures not very near $T_{\mathrm{c}}$, the mechanisms that play the most important role are (i) nonlinear electromagnetic response of the weak-link matrix (at low magnetic fields) [11, 35-37] and (ii) motion of Abrikosov vortices in the critical state (at $H>H_{\mathrm{c} 1 g}$ ) $[5,12,38]$.

In SC containing weak links, essentially two mechanisms of nonlinearity may come into play. Harmonic emission is expected when supercurrents are induced by the DC and MW magnetic fields in loops containing Josephson junctions. In this case, it is strictly related to the intrinsic nonlinearity of the Josephson current, $J_{\mathrm{c} j}[11,35]$. In addition, intergrain dynamics of Josephson fluxons may give rise to harmonic emission [36, 37, 39]. The results reported in figures 4 and 5 could arise from these mechanisms; however, no hysteresis is expected by none of the models describing these processes.

Magnetic hysteresis in the harmonic signals, as well as in the AC susceptibility, has been detected in ceramic SC at low frequencies $(100 \mathrm{~Hz}-100 \mathrm{kHz})$ [33-35], as well as at MW frequencies [13, 31]. At low frequencies, magnetic hysteresis in the same field range has been observed in the first, second and third harmonics, whose features strongly depend on the AC-driving-field intensity. The hysteretic behavior has been ascribed to the fact that, after the DC magnetic field has reached values large enough to allow Abrikosov fluxons penetrating the superconducting grains, the intergrain effective magnetic field in the decreasing-field branch is smaller than that in the increasing one in the virgin sample [33, 34], growing $J_{\mathrm{c} j}$. This process can justify the hysteresis we have detected in the harmonic response for $\mu_{0} H_{\max }>2 \mathrm{mT}$ (see figure 6), but it does not justify the hysteretic behavior of the SH signal at very low fields (very visible for $\mu_{0} H_{\max } \approx 0.2 \mathrm{mT}$ ), too small for creating intragrain vortices. Furthermore, we have not observed a noticeable variation in the hysteresis shape at different input power levels. 
In [31, 32], we suggested that the hysteretic behavior of the MW SH response in inhomogeneous SC can be related to the fact that both the above-mentioned nonlinear processes involving weak links come into play simultaneously. However, theoretical investigation performed by McDonald and Clem [39] on the electromagnetic response of Josephson junctions has shown that SH signals can be generated, whose sign is different whether fluxons nucleate or exit (i.e. the phase depends on the magnetic-field-sweep direction). In contrast, the phase of the even-harmonic signals arising from the nonlinearity of $J_{\mathrm{c} j}$ is not expected to depend on the fieldsweep direction, but only on the polarity of $H_{0}$ with respect to $H_{\mathrm{MW}}$. So, the interference of the two signals may give rise to the hysteretic behavior when $H_{\max } \geqslant H_{\mathrm{c} 1 j}$. The finding that we do not observe hysteresis in the TH signal at very low fields confirms this scenario; indeed, the phase of the odd-harmonic signals is expected to be independent of both the field-sweep direction and the $H_{0}$ polarity and no hysteresis comes out.

When taking into account all of all the above considerations, our results suggest that in the investigated sample $\mu_{0} H_{\mathrm{c} 1 j} \approx 0.2 \mathrm{mT}$ (the value of $H_{\max }$ at which the hysteresis appears in the $\mathrm{SH}$ signal) and $\mu_{0} H_{\mathrm{cl} g} \approx 2 \mathrm{mT}$ (the value of $H_{\max }$ at which the hysteresis appears in the $\mathrm{TH}$ signal). At higher DC fields, Abrikosov vortices penetrate the superconducting grains and additional mechanisms, due to dynamics of Abrikosov vortices, come into play, changing the features of both $\mathrm{SH}$ and $\mathrm{TH}$ versus $H_{0}$ curves. For $H_{\max }>$ $H_{\mathrm{clg}}$, the low-field signals in the decreasing-field branch reduces because some Josephson junctions are decoupled by the trapped flux.

Although the nonlinear processes occurring in weak links justify qualitatively the MW harmonic emission, a model able to quantitatively account for all the features of the harmonic signals has not yet been developed. Numerical calculations in the framework of the models elaborated to describe nonlinear emission by weak links [35-37] have shown that the intensity of the $\mathrm{SH}$ and $\mathrm{TH}$ signals, at their maximum value, are of the same order of magnitude. Furthermore, the TH versus $H_{0}$ curve is structured, with maxima and minima at low fields, while we observe a monotonically decrease with $H_{0}$. These results, and mostly the higher intensity of the $\mathrm{TH}$ signal, suggest that additional effects have to be taken into account to fully understand the nonlinear MW emission; so, further investigation is necessary.

\section{Conclusion}

We have built a tunable MW coaxial cavity resonator constituted by an outer $\mathrm{Cu}$ cylinder and an inner bulk $\mathrm{Bi}_{1.8} \mathrm{~Pb}_{0.26} \mathrm{Sr}_{2} \mathrm{Ca}_{2} \mathrm{Cu}_{3} \mathrm{O}_{10+x}$ cylinder. The cavity has been used to perform several kinds of measurements useful for investigating the MW response of the superconducting inner wire. In this paper, we have reported results of the MW surface resistance as a function of the frequency and the MW magnetic field. Moreover, by tuning the different modes of the cavity to make them resonant at exactly harmonic frequencies, we have detected the power emitted by the superconductor at the second- and third-harmonic frequency of the driving field. The intensity of the second- and third-harmonic signal has been investigated as a function of an external DC magnetic field at relatively low fields. Our results have been discussed in the framework of the models reported in the literature for the electromagnetic response of granular superconductors. All the results have been qualitatively accounted for considering processes occurring in weak links; however, a quantitative agreement, especially for the third-harmonic signal, has not been obtained. We have shown that this type of device provides a useful tool for a complete investigation of bulk superconductors of large dimension, both in the linear and nonlinear regimes, which can be conveniently used in bulkcavity filters. The advantage of using such a device is to measure different MW properties of the superconductor in the same excitation conditions. For example, the opportunity of investigating the second- and third-harmonic emission with the same device allowed us to highlight that third-harmonic emission is much more enhanced than second-harmonic one. This disagrees with the results expected from the models reported in the literature for nonlinear effects at low fields, arising from weak links. We would like to remark that by this device one can perform further measurements: for example, both the MW surface resistance and the harmonic signals can be measured as a function of the DC magnetic field at higher fields for investigating the Abrikosov-fluxon dynamics. Moreover, using a detection system working in a wider frequency range, higher harmonics can also be investigated.

\section{References}

[1] Lancaster M J 1997 Passive Microwave Device Applications of High-Temperature Superconductors (Cambridge: Cambridge University Press)

[2] Hein M 1999 High-Temperature Superconductor Thin Films at Microwave Frequencies (Springer Tracts of Modern Physics vol 155) (Heidelberg: Springer)

[3] Gallop J C 1997 Supercond. Sci. Technol. 10 A120

[4] Padamsee H 2001 Supercond. Sci. Technol. 14 R28

[5] Samoilova T B 1995 Supercond. Sci. Technol. 8259 and references therein

[6] Golosovsky M 1997 Proc. 1997 Workshop on RF Superconductivity p 295

[7] Nugyen P P, Oates D E, Dresselhaus G, Dresselhaus M S and Anderson A C 1995 Phys. Rev. B 516686

[8] Velichko A V, Lancaster M J and Porch A 2005 Supercond. Sci. Technol. 18 R24

Velichko A V, Cherpak N T, Izhyk E V, Kirichenko A Ya and Moroz A V 1998 Supercond. Sci. Technol. 11716

[9] Oates D E 1999 Microwave Superconductivity ed H Weinstock and M Nisenoff (Doldrecht: Kluwer Academic) pp 117-48

[10] Oates D E, Park S H and Koren G 2004 Phys. Rev. Lett. 93197001

[11] Ciccarello I, Guccione M and Li Vigni M 1989 Physica C 16139

[12] Agliolo Gallitto A, Ciccarello I, Guccione M and Li Vigni M 2003 Progress in Condensed Matter Physics ed G Mondio and L Silipigni (Bologna: Italian Physical Society) pp 93-104

[13] Agliolo Gallitto A, Bonsignore G, Di Gennaro E, Giunchi G, Li Vigni M and Manfrinetti P 2006 Microwave Opt. Technol. Lett. 482482

[14] Sridhar S and Kennedy W L 1988 Rev. Sci. Instrum. 59531

[15] Owliaei J, Sridhar S and Talvacchio J 1992 Phys. Rev. Lett. 693366 
[16] Fricano S, Bonura M, Agliolo Gallitto A, Li Vigni M, Klinkova L A and Barkowskii N V 2004 Eur. Phys. J. B 41313

[17] Bonura M, Di Gennaro E, Agliolo Gallitto A and Li Vigni M 2006 Eur. Phys. J. B 52459

[18] Dahm T, Scalapino D J and Willemsen B A 1999 J. Supercond. 12339

[19] Booth J C, Rudman D A and Ono R H 2003 IEEE Trans. Appl. Supercond. 13305

[20] Pandit H, Shi D, Babu N H, Chaud X, Cardwell D A, He P, Isfort D, Tournier R, Mast D and Ferendeci A M 2005 Physica C $\mathbf{4 2 5} 44$

[21] Leviev G I, Papikyan R S and Trunin M R 1991 Sov. Phys.-JETP 72201

[22] Delayen J R and Bohn C L 1989 Phys. Rev. B 405151

[23] Gallop J C, Radcliffe W J, Button T W and Alford N McN 1991 IEEE Trans. Magn. 271310

[24] Woodall P, Lancaster M J, Maclean T S M, Gough C E and Alford N McN 1991 IEEE Trans. Magn. 271264

[25] Piel H and Muller G 1991 IEEE Trans. Magn. 27854

[26] Alford N McN, Button T W, Peterson G E, Smith P A, Davis L E, Penn S J, Lancaster M J, Wu Z and Gallop J C 1991 IEEE Trans. Magn. 271510

[27] Alford N McN, Button T W and Opie D 1991 Supercond. Sci. Technol. 4433
[28] Halbritter J 1990 J. Appl. Phys. 686315

Halbritter J 1997 J. Supercond. 1091

[29] Hylton T L, Beasley M R, Kapitulnik A, Carini J P, Drabeck L and Grüner G 1989 IEEE Trans. Magn. 25810

[30] Herd J S, Oates D E and Halbritter J 1997 IEEE Trans. Appl. Supercond. 71299

[31] Agliolo Gallitto A, Bonsignore G, Giunchi G and Li Vigni M 2007 J. Supercond. Nov. Magn. 2013

[32] Agliolo Gallitto A, Guccione M and Li Vigni M 1998 Physica C 3098

[33] Sen D, Mitra A and Ghatak S K 1992 Supercond. Sci. Technol. 5 467-71

[34] Roy S B, Kumar S, Pradhan A K, Chaddah P, Prasad R, Soni N F and Adhikari K 1995 Supercond. Sci. Technol. 8155

[35] Lam Q H and Jeffries C D 1989 Phys. Rev. B 394772 Jeffries C D, Lam Q H, Kim Y, Kim C M, Zettl A and Klein M P 1989 Phys. Rev. B 3911526

[36] Ji L, Sohn R H, Spalding G C, Lobb C J and Tinkham M 1989 Phys. Rev. B 4010936

[37] Muller K H, MacFarlane J C and Driver R 1989 Physica C 158366

[38] Ciccarello I, Fazio C, Guccione M and Li Vigni M 1989 Physica C 159769

[39] McDonald J and Clem J R 1997 Phys. Rev. B 5614723 